\title{
Keratan Sulfate Restricts Neural Plasticity after Spinal Cord Injury
}

\author{
Shiro Imagama, ${ }^{1,2 \star}$ Kazuma Sakamoto, ${ }^{1 \star}$ Ryoji Tauchi, ${ }^{2}$ Ryuichi Shinjo, ${ }^{2}$ Tomohiro Ohgomori, ${ }^{1}$ Zenya Ito, ${ }^{1,2}$ \\ Haoqian Zhang, ${ }^{1,3}$ Yoshihiro Nishida, ${ }^{2}$ Nagamasa Asami, ${ }^{4}$ Sawako Takeshita, ${ }^{4}$ Nobuo Sugiura, ${ }^{5}$ Hideto Watanabe, ${ }^{5}$ \\ Toshihide Yamashita, ${ }^{6}$ Naoki Ishiguro, ${ }^{2}$ Yukihiro Matsuyama, ${ }^{2,7}$ and Kenji Kadomatsu ${ }^{1}$ \\ Departments of ${ }^{1}$ Biochemistry and ${ }^{2}$ Orthopedics, Nagoya University Graduate School of Medicine, Showa-ku, Nagoya 466-8550, Japan, ${ }^{3}$ Department of \\ Neurosurgery, University of California, San Francisco, California 94143-0110, ${ }^{4}$ Central Research Laboratories, Seikagaku Corporation, Higashiyamato, \\ Tokyo 207-0021, Japan, ${ }^{5}$ Institute for Molecular Science of Medicine, Aichi Medical University, Yazako, Nagakute, Aichi 480-1195, Japan, ${ }^{6}$ Department of \\ Molecular Neuroscience, Graduate School of Medicine, Osaka University, Suita, Osaka 565-0871, Japan, and ${ }^{7}$ Department of Orthopedics, Hamamatsu \\ University School of Medicine, Hamamatsu 431-3192, Japan
}

Chondroitin sulfate (CS) proteoglycans are strong inhibitors of structural rearrangement after injuries of the adult CNS. In addition to CS chains, keratan sulfate (KS) chains are also covalently attached to some proteoglycans. CS and KS sometimes share the same core protein, but exist as independent sugar chains. However, the biological significance of KS remains elusive. Here, we addressed the question of whether KS is involved in plasticity after spinal cord injury. Keratanase II (K-II) specifically degraded KS, i.e., not CS, in vivo. This enzyme digestion promoted the recovery of motor and sensory function after spinal cord injury in rats. Consistent with this, axonal regeneration/ sprouting was enhanced in K-II-treated rats. K-II and the CS-degrading enzyme chondroitinase $\mathrm{ABC}$ exerted comparable effects in vivo and in vitro. However, these two enzymes worked neither additively nor synergistically. These data and further in vitro studies involving artificial proteoglycans (KS/CS-albumin) and heat-denatured or reduced/alkylated proteoglycans suggested that all three components of the proteoglycan moiety, i.e., the core protein, CS chains, and KS chains, were required for the inhibitory activity of proteoglycans. We conclude that KS is essential for, and has an impact comparable to that of CS on, postinjury plasticity. Our study also established that KS and CS are independent requirements for the proteoglycan-mediated inhibition of axonal regeneration/sprouting.

\section{Introduction}

The CNS extracellular matrix (ECM) may play a role in the maintenance of the neuronal network by inhibiting axonal growth and suppressing the formation of additional inadequate synapses. Upon neuronal injury, disorganized production of proteoglycans in the ECM is initiated, leading to the inhibition of structural rearrangement of the neuronal network. Among proteoglycans, chondroitin sulfate proteoglycans (CSPGs) have received particular attention; dystrophic end balls end in the CSPG deposition

Received Sept. 29, 2010; revised Sept. 6, 2011; accepted 0ct. 1, 2011.

Author contributions: S.I., H.Z., Y.N., N.A., S.T., N.S., H.W., T.Y., N.I., Y.M., and K.K. designed research; S.I., K.S., R.T., and R.S. performed research; S.I., T.O., Z.I., and K.K. analyzed data; S.I. wrote the paper.

This work was supported in part by a Grant-in-Aid for Scientific Research on Innovative Areas (№. 23110002 to K.K.) from Ministry of Education, Culture, Sports, Science, and Technology (MEXT), Japan; by Grants-in-Aid for Scientific Research (Nos. 18390099 and 20390092 to K.K.) from MEXT, Japan; by the Ministry of Health, Labor, and Welfare of Japan (Health Sciences Research Grant on Comprehensive Research on Disability Health and Welfare, H21-012 to K.K.); and by funds from the Global Centers of Excellence program, MEXT, Japan, to Nagoya University. We thank K. Suzuki, Y. Kurahashi, and A. Tanaka (Seikagaku Corporation) for performing the stability test of K-Il and the HPLC analysis of sugar, M. Sawada (Nagoya University) for guidance with the primary culture of neurons, and N. Ozaki (Nagoya University) for guidance with the sensory tests. We also thank T. Natori (Yamanashi-Gakuin University) and M. lida, Y. Naito, S. Nakashima, N. Misawa, and Y. Miwa (Nagoya University) for their excellent technical assistance.

*S.I. and K.S. contributed equally to this work.

Correspondence should be addressed to Kenji Kadomatsu, Department of Biochemistry, Nagoya University Graduate School of Medicine, 65 Tsurumai-cho, Showa-ku, Nagoya 466-8550, Japan. E-mail: kkadoma@ med.nagoya-u.ac.jp.

DOI:10.1523/JNEUROSCI.5120-10.2011

Copyright $\odot 2011$ the authors $\quad 0270-6474 / 11 / 3117091-12 \$ 15.00 / 0$ area at the injury site (Davies et al., 1999; Grimpe and Silver, 2004; Silver and Miller, 2004; Tom et al., 2004), and CSPGs inhibit neurite outgrowth in vitro. The chondroitin sulfate (CS)degrading enzyme chondroitinase $\mathrm{ABC}(\mathrm{C}-\mathrm{ABC})$ promotes axonal regeneration after nigrostriatal tract transaction (Moon et al., 2001), collateral sprouting of spared fibers in the cuneate nucleus after cervical spinal cord injury (SCI) (Massey et al., 2006), and functional recovery after SCI (Bradbury et al., 2002). Thus, the CS chains of the CSPG moiety seem to be principal for the CSPG-mediated inhibition of structural rearrangement.

Proteoglycans consist of a core protein and covalently attached long sugar chains of repeating disaccharide units with sulfation, or so-called glycosaminoglycans (GAGs). Four sulfated glycosaminoglycans are known, i.e., CS, dermatan sulfate, heparan sulfate, and keratan sulfate (KS). Most proteoglycans carry a single glycosaminoglycan (such as a CSPG or KSPG), but a few proteoglycans, e.g., aggrecan (KS/ CSPG), have two types of glycosaminoglycan.

KS is expressed in the rodent roof plate of the spinal cord, and is induced after SCI and injury in the brain (Snow et al., 1990a; Cole and McCabe, 1991; Geisert et al., 1996; Jones and Tuszynski, 2002; Krautstrunk et al., 2002; Moon et al., 2002). In vitro digestion of KS restores neurite outgrowth on proteoglycan-coated substratum (Snow et al., 1990b; Powell et al., 1997). Thus, KS has been implicated in the regulation of axon guidance and/or axonal regeneration/sprouting. However, its biological impact on neu- 
ronal injuries and the underlying mechanisms have been poorly studied. We have recently found that mice deficient in the enzyme $\mathrm{N}$-acetylglucosamine 6-O-sulfotransferase-1 lose reactivity to the anti-KS antibody 5D4 in the brain, and show better axonal growth than wild-type mice after a cortical stab wound (Zhang et al., 2006). We have also found that the mice deficient in this enzyme show better motor function recovery and enhanced axonal regeneration/sprouting after SCI (Ito et al., 2010). However, as these knock-out mice lose $\mathrm{N}$-acetylglucosamine 6-Osulfotransferase- 1 in every cell in the body, critical questions remain to be answered, e.g., whether KS specifically works in the spinal cord, and how much impact KS has on functional disturbance. Furthermore, with regard to the functional redundancy between CS and KS, it is also an important question whether KS collaborates with CS, or works independently.

In the present study, we used keratanase II (K-II), which specifically degrades KS, and investigated the role of KS in postinjury plasticity. We found that KS and CS have a comparable impact on this form of plasticity. We also determined the structural basis of proteoglycan-mediated inhibition of neural plasticity.

\section{Materials and Methods}

Surgical procedure. Adult female Sprague Dawley rats weighing 200-230 g were used in the study of SCI. The animals were anesthetized with an intraperitoneal injection of ketamine $(100 \mathrm{mg} / \mathrm{kg})$ and xylazine $(10 \mathrm{mg} / \mathrm{kg})$. After Th9 laminectomy, we exposed the dura mater and induced injury using a force of $200 \mathrm{kdyn}$ using a commercially available SCI device (Infinite Horizon Impactor; Precision Systems and Instrumentation) that provided a consistent degree of spinal cord contusion injury. All injuries included the dorsal CST and dorsal gray matter. Immediately after the spinal cord contusion, we performed a Th12 partial laminectomy, inserted a thin silicone tube with an osmotic mini-pump into the subarachnoid cavity, and set the tube tip at the Th9 level under a surgical microscope. This tube was very soft and thin so that we could minimize damage to the spinal cord. The osmotic mini-pumps (200 $\mu \mathrm{l}$ of solution, $0.5 \mu \mathrm{l} / \mathrm{h}, 14 \mathrm{~d}$ delivery; Alzet pump model 2002 [Durect]) were filled with K-II (0.05 U/200 $\mu$ l; purified from Bacillus circulans) (Yamagishi et al., 2003), C-ABC (0.05 U/200 $\mu$ l) (Seikagaku), or saline (as a vehicle control). To determine an appropriate dose of $\mathrm{C}-\mathrm{ABC}$, we examined several doses and found that at the doses of 0.05, 0.1, and $1.0 \mathrm{U} / 200 \mu \mathrm{l}, \mathrm{C}-\mathrm{ABC}$ showed a comparable effect on motor function recovery, while the dose of $0.025 \mathrm{U} / 200 \mu \mathrm{l}$ showed a less potent effect (supplemental Fig. 1, available at www.jneurosci.org as supplemental material). Therefore, we used 0.05 $\mathrm{U} / 200 \mu \mathrm{l}$ of $\mathrm{C}-\mathrm{ABC}$ in this study.

The tube was sutured to the spinous process to anchor it in place, and the mini-pump was placed under the skin on the animal's back. Afterward, the muscles and skin were closed in layers. The bladder was compressed by manual abdominal pressure twice a day until bladder function was restored. Food was provided on the cage floor, and the rats had no difficulty reaching their water bottles. All animals were given antibiotics in their drinking water $[1.0 \mathrm{ml}$ of Bactramin (Roche) in $500 \mathrm{ml}$ of acidified water] for 2 weeks after SCI. We excluded the rats without complete paraplegia on the next day after operation in all groups as they were inappropriate for further evaluation. Although the number of such rats was very small ( $\sim 1$ of 70 rats after injury with our hands), we consistently found that rats without complete paraplegia showed a histology of incomplete injury of the spinal cord. However, no rats were excluded from any groups thereafter, with the exception of rats that had died. We performed postmortem histological measurement and found that all the dead rats had complete severe injury in the spinal cord (data not shown). Therefore, it was not likely that rats with serious spinal shock but minor injury were involved in the experiments in the current study. The number of rats undergoing operation and the number of rats evaluated in each experiment are summarized in Table 1. All animals were treated and cared for in accordance with the Nagoya University School of Medicine Guidelines pertaining to the treatment of experimental animals.

Basso, Beattie, and Bresnahan open field locomotor test. The recovery of hindlimb motor function was assessed by determining the Basso, Beattie,
Table 1. Number of rats undergoing operation and evaluated in each experiment

\begin{tabular}{lll}
\hline & \multicolumn{2}{l}{ Rats } \\
\cline { 2 - 3 } Models & Number used for operation & Final number evaluated \\
\hline EXP1: concentration (Fig. 2D,E) & & \\
K-II 0.05 U & 10 & 7 \\
K-II 0.005 U (1/10) & 10 & 5 \\
K-II 0.000025 U (1/2000) & 10 & 6 \\
K-II (heat-denatured) & 10 & 5 \\
Vehicle & 10 & 7 \\
EXP2: functional recovery (Fig. 2A,B) & & \\
K-II 0.05 U & 12 & 10 \\
C-ABC & 12 & 7 \\
Vehicle & 12 & 10 \\
EXP3: functional recovery (Fig. 2F) & & \\
Vehicle & 12 & 9 \\
C-ABC & 12 & 7 \\
K-II & 12 & 11 \\
K-II+C-ABC & 12 & 7 \\
EXP4: MEP (Fig. 2C) & & \\
Sham & 10 & 8 \\
Vehicle & 10 & 9 \\
C-ABC & 10 & 5 \\
K-II & 10 & 7 \\
K-II+C-ABC & 10 & 7 \\
\hline
\end{tabular}

EXP, Experiment.

and Bresnahan (BBB) scores (Basso et al., 1995). The results were quantified in a blinded manner by two observers.

$\%$ Grip test. Paw placement for each limb on the grid bar was assessed as the animal walked on a plastic-coated wire mesh grid $(50 \mathrm{~cm}$ length $\times 33$ $\mathrm{cm}$ width $\times 20 \mathrm{~cm}$ height, with $2.5 \times 2.5 \mathrm{~cm}$ openings) for $3 \mathrm{~min}$. Steps in which the paw gripped the grid bar and supported the animal's weight were counted as correct. The number of correct paw placements was expressed as a percentage of the total steps. The percentage of correct paw placements was calculated for each hindlimb and averaged.

Touch test. A touch test was performed preoperatively and then weekly after the operation. Rats were habituated for $30 \mathrm{~min}$ in elevated clear plastic cages with wire mesh grid floors. Graded von Frey hair ( $\mathrm{vFH})$ monofilaments were applied to the plantar surface of the foot, $\sim 1 \mathrm{~cm}$ posterior to the footpad of the middle phalange with the up-down method using procedures described in detail previously (Hutchinson et al., 2004).

Testing began with $15.14 \mathrm{~g} \mathrm{vFH}$ applied to the hindpaw and continued until 10 trials per hindpaw were completed. Rats were given a food reward throughout testing to prevent visual recognition of the application of the monofilament. A trial was discarded and reperformed if $\mathrm{vFH}$ application lifted the paw, producing proprioceptive rather than tactile input. A positive response occurred when the paw was briskly withdrawn from the monofilament. The lowest gram force that produced withdrawal was designated as the response threshold. The thresholds of the bilateral hindpaws were averaged. It was previously determined that the average $\mathrm{vFH}$ hindpaw threshold in normal rats is $60 \mathrm{~g}$; in the present study, therefore, we preoperatively excluded rats with a vFH threshold higher than this value. The examiners were blind to group assignment during the $\mathrm{vFH}$ testing.

Tail-immersion test. The tail-immersion test was used to determine the somatic thermo-threshold of rats preoperatively and then weekly after the operation. Briefly, rats were gently lifted from their home cages and taken to the test room, where the withdrawal latency of the tail to a temperature stimulus was measured by immersing the tail in a $55 \pm$ $0.5^{\circ} \mathrm{C}$ water bath. The cutoff time was set at $8 \mathrm{~s}$ to avoid skin damage. Rats underwent this test three times at intervals of $15 \mathrm{~min}$. The measurements were averaged to obtain the withdrawal latency time in seconds. Between sessions, the rats were returned to their home cages. Based on the results, we preoperatively excluded rats with prolonged withdrawal latency. Examiners were blind to the group assignment during the tail-immersion test.

Western blotting. The 10-mm-long sections of the injured spinal cord at 1 week after SCI were dissected and homogenized in PBS including 1\% 
Table 2. Purification of keratanase II

\begin{tabular}{llll}
\hline \multirow{2}{*}{ Step } & \multicolumn{2}{l}{ Enzyme activity (units) } \\
\cline { 2 - 4 } & BcKeratanase & Chondroitinase & $\alpha$-Galactosidase \\
\hline Cell extract & 38.000 & 7.000 & 12.000 \\
Salting out & 33.060 & 3.500 & 3.960 \\
Desalting & 29.260 & 3.150 & 3.600 \\
DEAE-cellulose & 20.900 & 0.013 & 2.400 \\
Phenyl-sepharose & 20.900 & 0.000 & 0.000 \\
\hline
\end{tabular}

Keratanase unit: One unit will liberate $1.0 \mu \mathrm{mol}$ of reducing sugar as 2-acetamido-2-deoxy-D-glucose from keratan sulfate per minute at $\mathrm{pH} 6.0$ at $37^{\circ} \mathrm{C}$. Chondroitinase unit: One unit will liberate $1.0 \mu \mathrm{mol}$ of 2-acetamido-2-deoxy3-0-( $\beta$-D-gluc-4-ene-pyranosyluronic acid)-6-0-sulfo-D-galactose from chondroitin sulfate $C$ per minute at $\mathrm{pH} 8.0$ at $37^{\circ} \mathrm{C} . \alpha$-Galactosidase unit: One unit will hydrolyze $1.0 \mu \mathrm{mol}$ of $p$-nitrophenyl- $\alpha$-D-galactopyranoside per minute at $\mathrm{pH} 6.5$ at $37^{\circ} \mathrm{C}$.

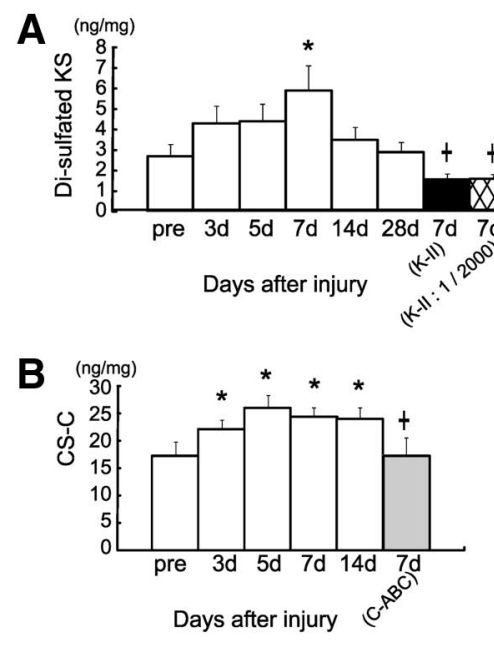

D

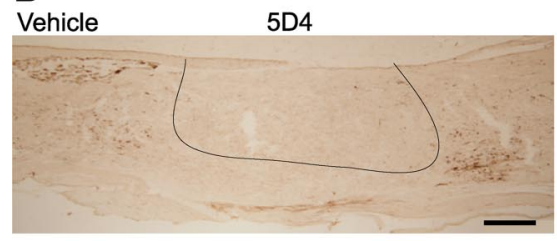

$\mathrm{C}-\mathrm{ABC}$

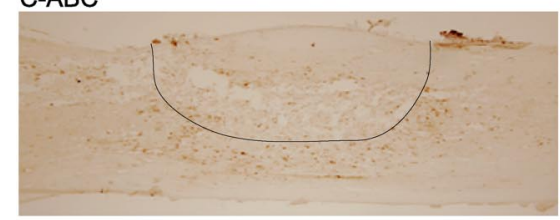

$\mathrm{K}-\mathrm{II}$

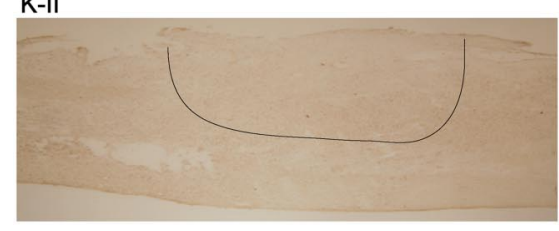

C

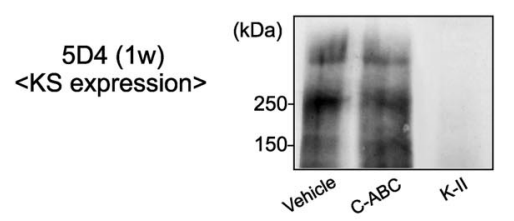

MC21C (1w) $<$ CS expression>

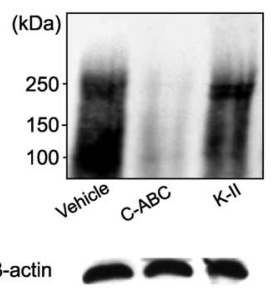

$\mathbf{E}$

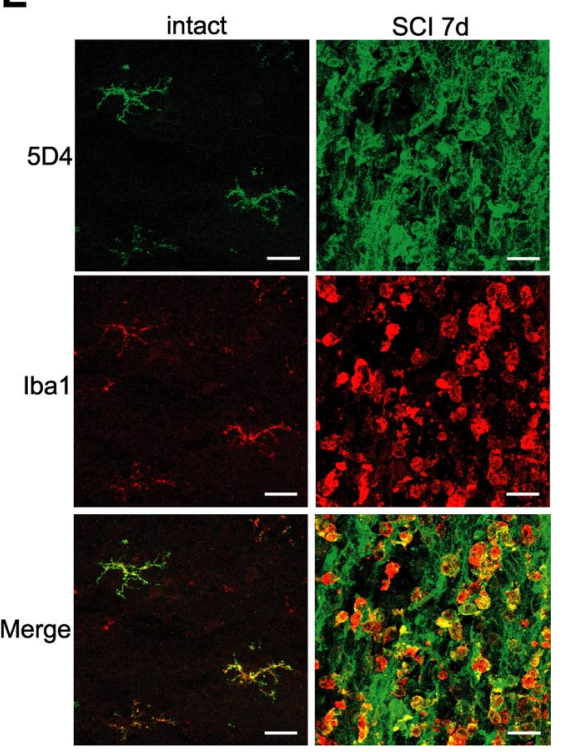

Figure 1. Specific degradation of KS by K-II.A, Amounts of disulfated KS after SCI. A $10 \mathrm{~mm}$ fragment of the injured spinal cord was subjected to the analysis. Data represent the means \pm SEM. ${ }^{*} p<0.05$ versus the preinjured specimen (pre). ${ }^{+} p<0.05$ versus $7 \mathrm{~d} ; n=5$ in each group. K-II, $0.05 \mathrm{U}$ of K-II was administered to the spinal cord; K-II 1/2000, $0.0000025 \mathrm{U}$ of K-II was administered to the spinal cord. $\boldsymbol{B}$, Amounts of $\mathrm{CS}-\mathrm{C}$ after $\mathrm{SCl} .{ }^{*} p<0.05$ versus pre; ${ }^{+} p<0.05$ versus $7 \mathrm{~d} ; n=6$ in each group. $C-A B C, 0.05 \cup$ of $C-A B C$ was administered to the spinal cord. C, Rats were treated with K-II or C-ABC for 1 week after spinal cord injury. A $10 \mathrm{~mm}$ specimen of the injured spinal cord was subjected to Western blot analysis for 5D4-reactive KS, MC21C-reactive CS, and $\beta$-actin. $\boldsymbol{D}, 5 \mathrm{D} 4$-reactive $\mathrm{KS}$ was visible in the penumbra region in vehicle- and C-ABC-treated rats, but not in K-II-treated rats. These rats were treated with the indicated enzyme for 1 week after spinal cord injury. Scale bars, $500 \mu \mathrm{m}$. All photos are of sagittal sections with the left side rostral. $\boldsymbol{E}$, Double staining for KS (5D4) and microglia (Iba1). The left panels show the intact spinal cord, and the right panels show the spinal cord 1 week after injury. Most 5D4-reactive cells overlapped Iba1-positive cells, which indicated that KS was mainly expressed by microglia. The left images showed ramified microglia expressing 5D4-KSPG. The right images show that 5D4-KSPG expression is seen on characteristic cellular profiles with short processes and a less ramified morphology after SCI. Scale bars, $20 \mu \mathrm{m}$.
Triton X-100 and protease inhibitors solution (Sigma). Samples of the supernatant fraction were collected after centrifuging at $10,000 \times g$ for 30 min. Thirty microliters of each sample was applied and separated by trocellulose membranes. Blots were blocked with $5 \%$ fat-free dry milk in Seikagaku) in PBS containing $0.3 \%$ Triton X-100, washed, and then incubated with a second antibody, HRP-conjugated goat anti-mouse IgM (1/5000; Southern Biotechnology), at room temperature for $60 \mathrm{~min}$. Anti- $\beta$-actin antibody (1/100,000; Sigma) was also used as indicated. Bound antibodies were visualized with an ECL-plus Western blotting detection kit (GE Healthcare).

Immunohistochemistry and immunocytochemistry. After terminal anesthesia by ether hyperaspiration, rats were perfused transcardially with buffered $4 \%$ paraformaldehyde. The spinal cords and brains were removed, postfixed overnight, and then cryoprotected in buffered $30 \%$ sucrose the next night. Tissues were cut into $20 \mu \mathrm{m}$ sections with a cryostat and mounted on glass slides. The sections were blocked in PBS containing 3\% BSA and 5\% normal mouse serum for staining of biotinconjugated anti-KS 5D4 or blocked in PBS containing 1\% BSA and 10\% normal goat serum for other immunohistochemistry. The sections were then incubated with the primary antibodies to KS (5D4; Seikagaku), Ibal (Wako Pure Chemical Industries), GAP-43 (Millipore), serotonin (5-HT, Immunostar), type IV collagen (LSL), and GFAP (Sigma). After rinsing, the sections were incubated with the secondary antibody for $60 \mathrm{~min}$ at room temperature: $\mathrm{Cy} 3-$ or Cy2-conjugated streptavidin (Jackson ImmunoResearch), Cy3-conjugated goat anti-rabbit IgG (Zymed Laboratories), Cy3-conjugated goat anti-mouse IgM (Jackson ImmunoResearch), FITC-conjugated goat anti-rat IgG (Sigma), and Alexa Fluor 488 goat anti-mouse IgG (Invitrogen). Sections were then rinsed, mounted with FluorSave (Calbiochem), and examined by confocal microscopy (MRC 1024; Bio-Rad Laboratories).

Morphometry. The extent of axonal outgrowth of each wound area was assessed by counting signals visualized on staining with GAP-43 and 5-HT using serial sagittal $20 \mu \mathrm{m}$ sections. Morphometry analysis was performed using a computer-driven microscope stage (MetaMorph Offline, version 6.3r ${ }^{2}$; Molecular Devices Corporation). In all sagittal sections shown here, the left side is rostral.

Anterograde labeling of the corticospinal tract. Eight weeks after injury, descending corticospinal tract (CST) fibers were labeled with biotin-dextran amine (BDA; 10\% in saline, 3.5 $\mu l$ per cortex, molecular weight 10,000 ; Invitrogen) injected under anesthesia into the left and right motor cortices (coordinates, $2 \mathrm{~mm}$ posterior and $2 \mathrm{~mm}$ lateral to the bregma; 1.5 $\mathrm{mm}$ depth). For each injection, $0.25 \mu \mathrm{l}$ of BDA was delivered for a period of $30 \mathrm{~s}$ via a $15-20$ $\mu \mathrm{m}$ inner diameter glass capillary attached to a microliter syringe (ITO Corporation). Two weeks after BDA injection, the animals were killed by perfusion with PBS followed by $4 \%$ paraformaldehyde. The spinal cords were dissected, postfixed overnight in the same fixa- 
tives, and cryopreserved in $30 \%$ sucrose in PBS. A $20 \mathrm{~mm}$ length of spinal cord, $10 \mathrm{~mm}$ rostral and $10 \mathrm{~mm}$ caudal to the lesioned site, was embedded in Tissue-Tek OCT. These blocks were sectioned in the transverse plane $(25 \mu \mathrm{m})$. Sections were incubated in PBS with $0.3 \%$ Triton $\mathrm{X}$ for $4 \mathrm{~h}$ and then incubated for $2 \mathrm{~h}$ with Alexa Fluor 488-conjugated streptavidin (1:400; Invitrogen) in PBS with $0.05 \%$ Tween 20. We then took serial cross sections of the spinal cord and quantitatively analyzed the distribution of the axons. Degrees of BDA uptake were assessed by counting the total number of fibers in the cross section $10 \mathrm{~mm}$ rostral to the lesioned site, where the CST was intact. For quantification of the number of labeled corticospinal axons $10 \mathrm{~mm}$ caudal to the lesion site, the number of labeled fibers was counted in the gray matter, the dorsal CST area (normal locations of the dorsal CST), or the white matter except for the dorsal CST area, and divided by the number of labeled corticospinal axons 10 $\mathrm{mm}$ above the lesion. The labeled fibers were counted using MetaMorph software (Molecular Devices Corporation). Light intensity and thresholding values were maintained at constant levels for all analyses.

Motor-evoked potential. In terminal electrophysiological experiments, after an intraperitoneal injection of ketamine $(100 \mathrm{mg} / \mathrm{kg})$, short trains of five square-wave stimuli of 0.5 ms duration at interstimulus intervals of $2 \mathrm{~ms}$ were delivered through the epidural electrode catheter at a point $10 \mathrm{~mm}$ rostral from the epicenter. The active electrode was placed in the muscle belly, and the reference electrode was placed near the distal tendon of the muscle in each gastrocnemius. The ground electrode was placed subcutaneously between the epidural electrode and the recording electrodes. The onset latency was measured as the length of time in milliseconds between the stimulus and the onset of the first wave. The duration was also measured as the length of time in milliseconds between the waves. One hundred responses were averaged and stored for off-line analysis.

Spinal cord-evoked potential after stimulation to the spinal cord. For sensory assessment, we performed spinal cord-evoked potential after stimulation to the spinal cord (sp-SCEP), which recognized extrapyramidal pathways including dorsal column axons (Sutter et al., 2007; Tamaki and Kubota, 2007). Stimulations were delivered through the epidural electrode catheter at a point $10 \mathrm{~mm}$ caudal from the epicenter and measured at a point $10 \mathrm{~mm}$ rostral from the epicenter. The ground electrode was placed subcutaneously between the epidural electrodes. The onset latency and peak latency were measured as the length of time in milliseconds between the stimulus and the onset and the peak of the first wave, respectively. A total of 100 responses were averaged and stored for off-line analysis of the latency.

Measurement of CS and KS. After freeze-drying, each specimen was digested with $0.7 \mathrm{ml}$ of $2.5 \%$ actinase E (Kaken Pharmaceutical Corporation) at $55^{\circ} \mathrm{C}$ for $24 \mathrm{~h}$. The digest was then kept at $100^{\circ} \mathrm{C}$ for $5 \mathrm{~min}$ and centrifuged at $3000 \mathrm{rpm}$ for $10 \mathrm{~min}$. The CS concentration was determined according to the description of Shinmei et al. (1992) with some modifications. A $0.2 \mathrm{ml}$ aliquot of the supernatant was digested with 250 $\mathrm{mU}$ of C-ABC (Seikagaku) and $25 \mathrm{mU}$ of chondroitinase AC-II (Seikagaku) in $20 \mathrm{~mm}$ Tris- $\mathrm{HCl}$ buffer, $\mathrm{pH} 8$, at $37^{\circ} \mathrm{C}$ for $2 \mathrm{~h}$. The sample was $11 \mathrm{~K}-1 \mathrm{I} ; n=7 \mathrm{~K}-\mathrm{II}+\mathrm{C}-\mathrm{ABC})$.
B

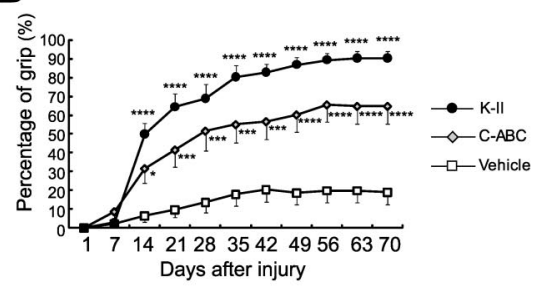

Days after injury
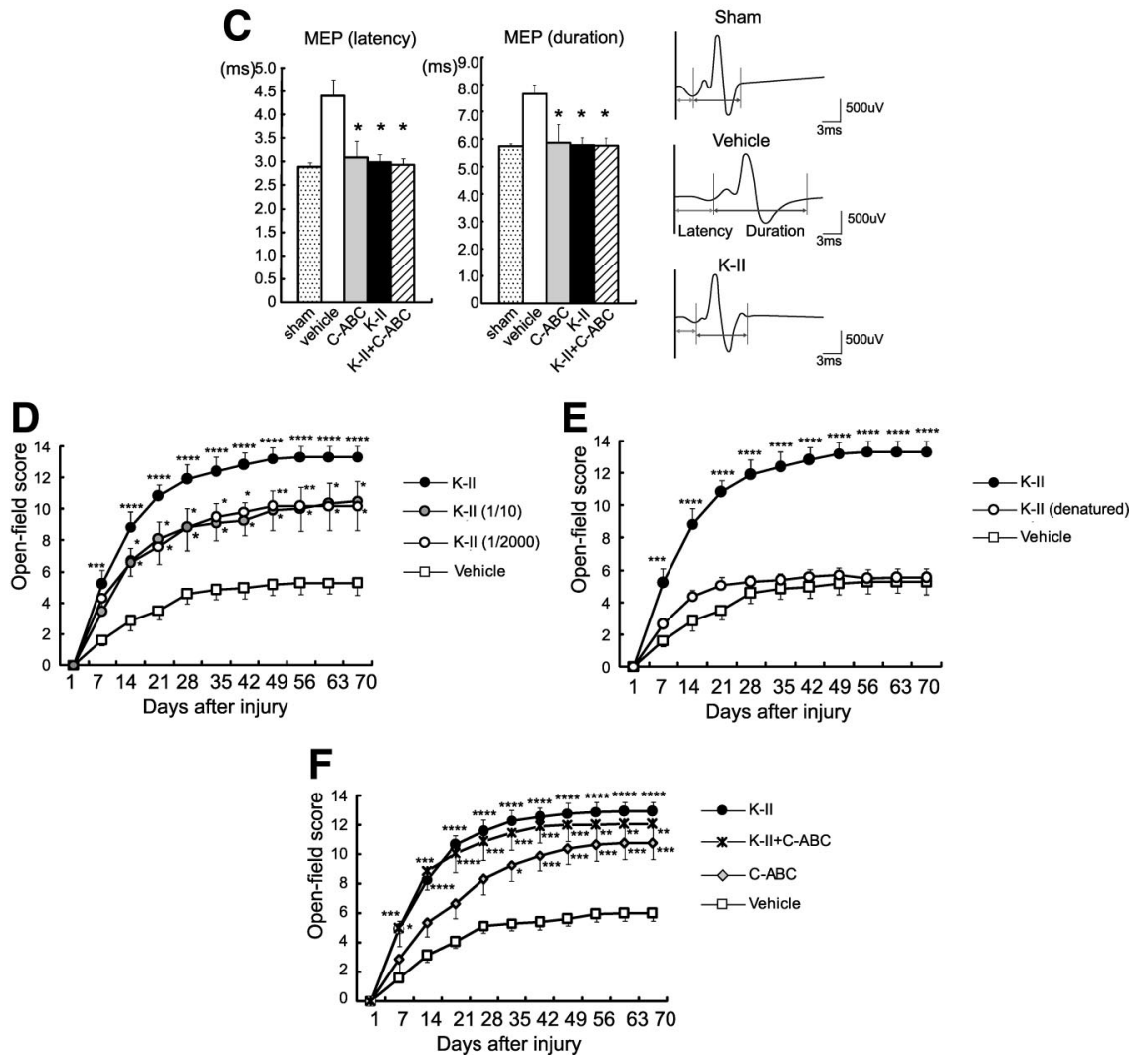

Figure 2. Motor function recovery by K-II after SCI. A, B, The BBB score and \%grip test data after SCI. Recovery was significantly better in the $\mathrm{K}-\mathrm{Il}$ and $\mathrm{C}-\mathrm{ABC}$ treatment groups than in the vehicle-administered control. Data represent the means \pm SEM. ${ }^{*} p<$ $0.05 ;{ }^{* * *} p<0.005 ;{ }^{* * *} p<0.001$ versus the vehicle control ( $n=10 \mathrm{~K}-11 ; n=7$ C $-\mathrm{ABC} ; n=10$ Vehicle). C, Electrophysiologic tests using MEP also showed functional recovery in the treatment groups at 8 weeks after SCI. Data represent the means \pm SEM. $p<0.05$ versus the vehicle control $(n=8$ Sham; $n=9$ Vehicle; $n=5$ C $-\mathrm{ABC} ; n=7 \mathrm{~K}-\mathrm{II} ; n=7 \mathrm{~K}-\mathrm{II}+\mathrm{C}-\mathrm{ABC})$. D. Motor function ren restored motor function at concentrations of $0.005 \mathrm{U}(1 / 10)$ and $0.0000025 \mathrm{U}(1 / 2000)$. Data represent the means \pm SEM. ${ }^{*} p<$ 7 SEM. ${ }^{* *} p<0.005 ;{ }^{* * *} p<0.001$ versus the vehicle control ( $n=7 \mathrm{~K}-$ II; $n=5$ Heat-denatured K-II; $n=7$ Vehicle). $\boldsymbol{F}$, The combination of K-II and C-ABC did not show additive or synergistic effects on motor function (BBB score). Data represent the means \pm SEM. ${ }^{*} p<0.05 ;{ }^{* *} p<0.01 ;{ }^{* * *} p<0.005 ;{ }^{* * * *} p<0.001$ versus the vehicle control $(n=9$ Vehicle; $n=7$ C $-A B C ; n=$

ultrafiltrated using a Nanosep centrifugal device (molecular size cutoff 10,000 ; PALL), and the filtrate, which contained the unsaturated disaccharides $\Delta$ di-0S, $\Delta$ di- 4 S, and $\Delta$ di-6S derived from CS, was analyzed by HPLC.

The KS concentration was measured according to the method of Shinmei et al. (1992) with some modifications. A $0.15 \mathrm{ml}$ aliquot of the supernatant was digested with $1 \mathrm{mU}$ of K-II (Seikagaku) in $20 \mathrm{~mm}$ sodium acetate buffer, $\mathrm{pH} 6$, at $37^{\circ} \mathrm{C}$ for $3 \mathrm{~h}$. The sample was then ultrafiltrated using a Nanosep centrifugal device, and the filtrate, which contained the saturated disaccharides $\beta$-galactosyl-(1-4)-6-O-sulfo- $N$-acetylglucosamine and $\beta$-6-O-sulfogalactosyl-(1-4)-6-O-sulfo- $\mathrm{N}$-acetylglucosamine derived from $\mathrm{KS}$, was analyzed by HPLC.

The HPLC system used in this study consisted of a Model 2000 highperformance chromatograph (Jasco) and a stainless steel column packed 
A
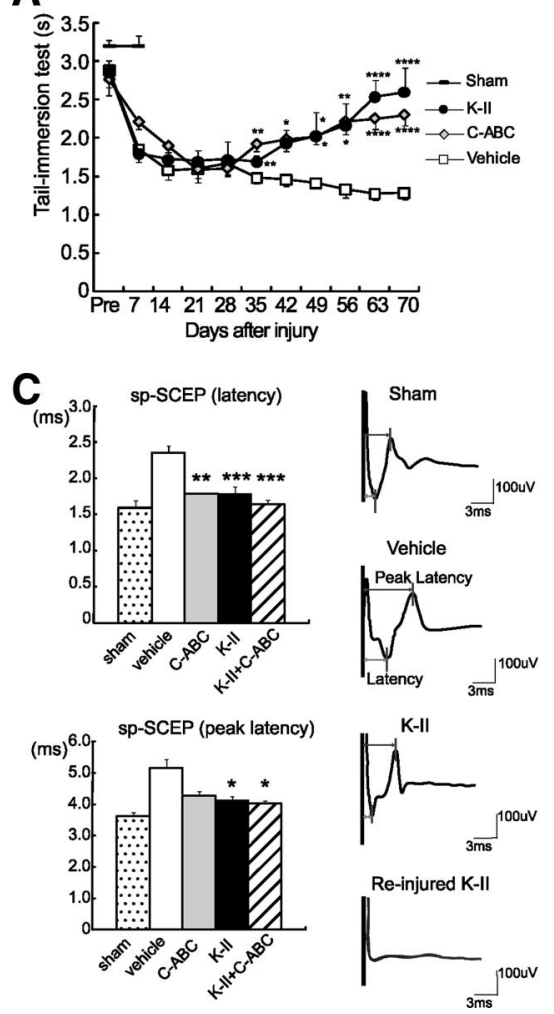

Figure 3. Sensory function recovery by $\mathrm{K}-\mathrm{Il}$ after $\mathrm{SCl}$. $\boldsymbol{A}$, The tail-immersion test indicated that there was little allodynia in the K-II- and C-ABC-treated rats. Data represent the means \pm SEM. ${ }^{*} p<0.05 ;{ }^{* *} p<0.01$; ${ }^{* * * *} p<0.001$ versus the vehicle control ( $n=7 \mathrm{Sham} ; n=5 \mathrm{~K}-1 \mathrm{l} ; n=7 \mathrm{C}-\mathrm{ABC} ; n=9$ Vehicle). $\boldsymbol{B}$, The touch test also indicated that there was little allodynia in the $\mathrm{K}-\mathrm{Il}-$ and C-ABC-treated rats. Data represent the means \pm SEM. ${ }^{*} p<0.05 ;{ }^{* * *} p<0.005$ versus the vehicle control $(n=7$ Sham; $n=$ $5 \mathrm{~K}-\mathrm{II} ; n=7$ C-ABC; $n=9$ Vehicle). C, Electrophysiologic tests using sp-SCEP showed not only motor functional recovery but also sensory recovery in the treatment groups at 8 weeks after $\mathrm{SCl}$. The reinjury of K-II-treated rats lost the recovered response of sp-SCEP. Data represent the means \pm SEM. ${ }^{*} p<0.05 ;{ }^{* *} p<0.01 ;{ }^{* * *} p<0.005$ versus the vehicle control $(n=8$ Sham; $n=$ 7 Vehicle; $n=5$ C-ABC; $n=6 \mathrm{~K}-11 ; n=5 \mathrm{~K}-\| \mathrm{H}+\mathrm{C}-\mathrm{ABC})$. $\boldsymbol{D}$, The combination of K-II and C-ABC did not show additive or synergistic effects on sensory function (tail-immersion test). Data represent the means \pm SEM. ${ }^{*} p<0.05 ;{ }^{* *} p<0.01 ;{ }^{* * *} p<0.005$; ${ }^{* * *} p<0.001$ versus the vehicle control ( $n=8$ Sham; $n=9$ Vehicle; $n=7$ C-ABC; $\left.n=11 \mathrm{~K}-\mathrm{Il} ; n=7 \mathrm{~K}-\mathrm{II}+\mathrm{C}-\mathrm{ABC}\right)$.

with polyamine-bound silica (YMC gel PA-120; YMC). The disaccharides in each sample were eluted with a gradient of $0-100 \mathrm{~mm}$ sodium sulfate. To the eluant from the column was added $100 \mathrm{~mm}$ sodium tetraborate buffer, $\mathrm{pH} 9$, containing $1 \% 2$-cyanoacetamide. The mixture was thermostated at $145^{\circ} \mathrm{C}$, and the effluent was monitored by a fluoromonitor.

Cell culture. Sprague Dawley rats at postnatal days 7-9 were killed and their cerebella were collected. The meninges were carefully removed with fine forceps, and the tissues were minced and digested using a Papain Dissociation System (Worthington). Dissociated cells were applied to a $35 / 60 \%$ two-step Percoll gradient and centrifuged at $3000 \times g$ for $15 \mathrm{~min}$. Cerebellar granule neurons at the interface were collected. Cells were suspended in Neurobasal medium (Invitrogen) supplemented with 2\% B27 (Invitrogen), $2 \mathrm{~mm}$ glutamine, an additional $20 \mathrm{mM} \mathrm{KCl}, 50 \mathrm{U} / \mathrm{ml}$ penicillin, and $50 \mu \mathrm{g} / \mathrm{ml}$ streptomycin.

Substrate preparation. Four-well chamber slides (Nunc) were coated with $20 \mu \mathrm{g} / \mathrm{ml}$ poly-L-lysine (PLL) (Sigma) and left overnight at $4^{\circ} \mathrm{C}$. They were then coated with the indicated substrates and left for $4 \mathrm{~h}$ at $37^{\circ} \mathrm{C}$. If indicated, proteoglycans or aggrecan were treated with 200 $\mathrm{mU} / \mathrm{ml} \mathrm{C}-\mathrm{ABC}, 500 \mathrm{mU}$ keratanase, and $5 \mathrm{mU} / \mathrm{ml} \mathrm{K}$-II derived from Bacillus sp. Ks36 or $5 \mathrm{mU}$ endo- $\beta$-galactosidase (all from Seikagaku) in $\mathrm{PBS}$ at $37^{\circ} \mathrm{C}$. Other substrate materials included poly-L-ornithine, aggrecan, and MAG (Sigma), Nogo and OMgp (R\&D Systems), and KS and CS-C (Seikagaku).

Neurite outgrowth assay. Cerebellar granule neurons were seeded onto four-well chamber slides at $2.0 \times 10^{5} /$ well. Twenty-four hours after seeding, the neurons were fixed with $4 \%$ paraformaldehyde/PBS and stained with anti-neuron-specific $\beta$-tubulin (Covance) to visualize the neurites. Neurite lengths were measured from at least 100 neurons per condition from duplicate wells, and quantified as described previously (Sivasankaran et al., 2004).

For reducing/alkylating proteoglycans, $5 \mu \mathrm{g}$ of proteoglycans were suspended in $10 \mathrm{ml}$ of 50 $\mathrm{mM}$ ammonium bicarbonate/PBS and reduced with $20 \mathrm{~mm}$ DTT for $60 \mathrm{~min}$ at $37^{\circ} \mathrm{C}$. Then proteoglycans were alkylated with $10 \mathrm{~mm}$ iodoacetamide for $30 \mathrm{~min}$ at room temperature under dark condition. The proteoglycan solution was dialyzed against PBS for overnight and subjected to neurite outgrowth assay.

Preparation of GAG-BSA. CS-BSA was prepared as reported previously (Pumphrey et al., 2002) with minor modifications. Briefly, $20 \mathrm{mg}$ of CS and $13 \mathrm{mg}$ of BSA were linked using $\mathrm{NaBH}_{3} \mathrm{CN}$. CS-BSA was purified using anionexchange chromatography (Q-Sepharose; GE Healthcare) followed by gel-filtration chromatography (Superose6 HR10/300; GE Healthcare) to remove free-BSA and free-CS, respectively. The protein concentration and KS concentration were quantified using a BCA Protein Assay Kit (Pierce) and HPLC analysis, respectively.

Because KS had an oligopeptide at its reducing end, we performed cross-linking between this peptide and BSA. Briefly, amino residues of this peptide were succinylated to inhibit self-crosslinking. Fifty-two milligrams of BSA and $20 \mathrm{mg}$ of succinylated KS were cross-linked using $\mathrm{N}$-hydroxysuccinimide (Pierce) and 1-ethyl-3[3-dimethylaminopropyl]carbodiimide hydrochloride (Pierce). KS-BSA was purified using anion-exchange chromatography (Q-Sepharose; GE Healthcare) followed by gel-filtration chromatography (Superose6 HR10/300; GE Healthcare) to remove free-BSA and free-KS, respectively. The protein concentration and KS concentration were quantified using a BCA Protein Assay Kit (Pierce) and a Sulfated Glycosaminoglycan Quantification Kit (Seikagaku), respectively.

CS/KS-BSA was prepared by linking KS to CS-BSA.

Statistical analysis. We performed statistical analysis with an unpaired two-tailed Student's $t$ test for single comparisons and one-way ANOVA for multiple comparisons. For the BBB score, \%grip test, touch test, and tail-immersion test, we used repeated-measures ANOVA and Tukey's test. In all statistical analyses, significance was accepted at $p<0.05$.

\section{Results}

\section{In vitro and in vivo degradation of KS by K-II}

We purified K-II from B. circulans as previously described (Yamagishi et al., 2003). Although the extracts of B. circulans showed both keratanase and chondroitinase activities, our stepwise protocol completely abolished the chondroitinase activity and purified K-II (Table 2). Furthermore, it is known that the purified enzyme does not digest hyaluronic acid, heparan sulfate, or heparin (Yamagishi et al., 2003). This enzyme is thermo-stable; indeed, it retained its enzymatic activity for at least $120 \mathrm{~h}$ at $37^{\circ} \mathrm{C}$ $(100 \pm 1.606 \%$ at $0 \mathrm{~h}$ vs $91.9 \pm 4.014 \%$ at $120 \mathrm{~h})$. Therefore, we decided to use this enzyme for in vivo experiments.

We asked whether K-II worked in vivo. To this end, we inflicted contusion injuries using a force of $200 \mathrm{kdyn}$ in the rat spinal cord at the ninth thoracic vertebral level. This contusion model completely destroyed the dorsal columns and dorsal CST 
A

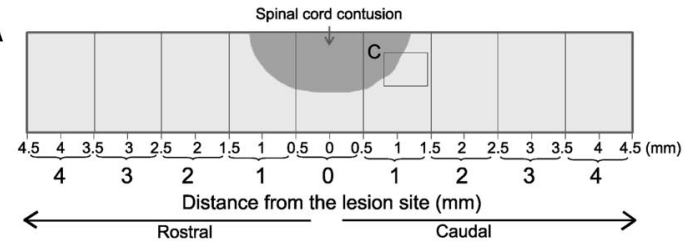

B
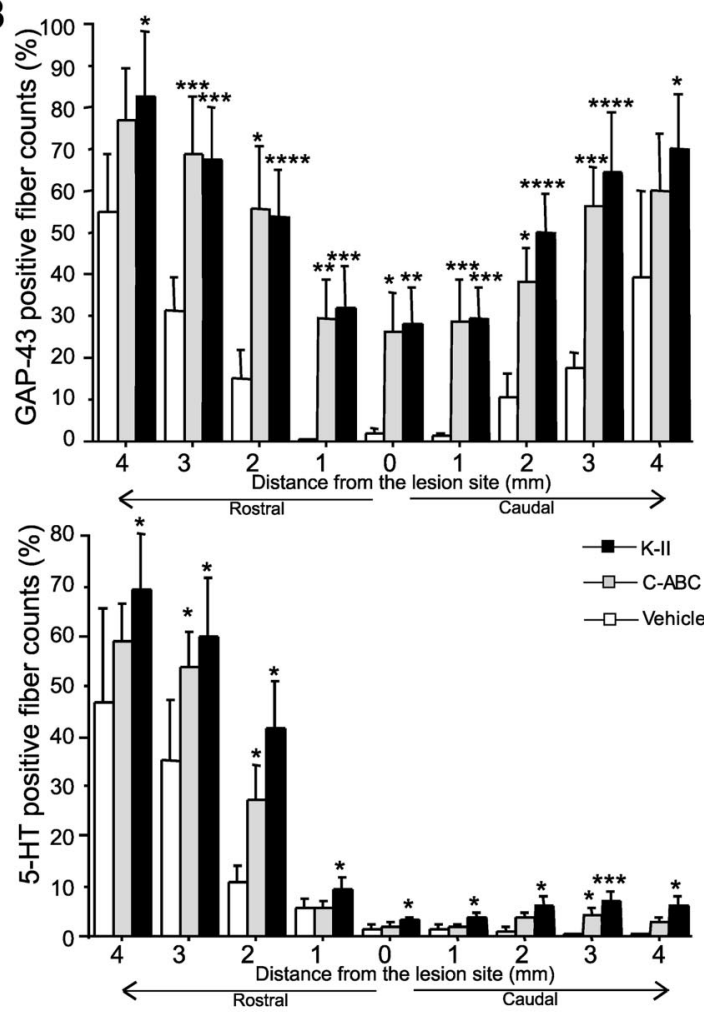

D

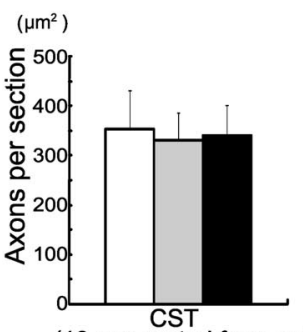

(10 $\mathrm{mm}$ rostral from epicenter)

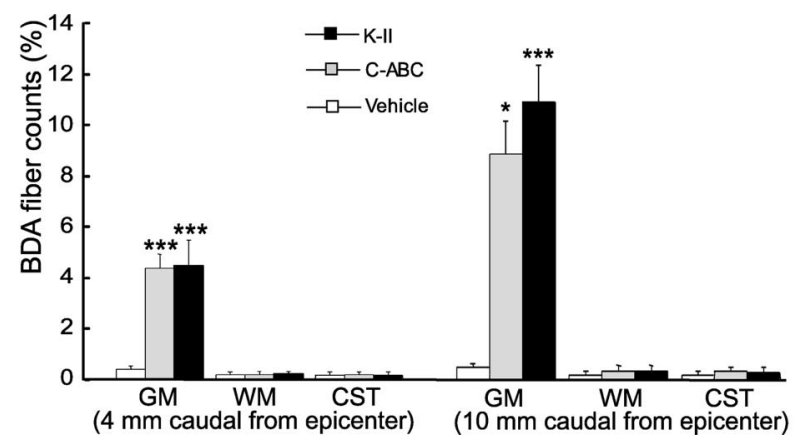

C
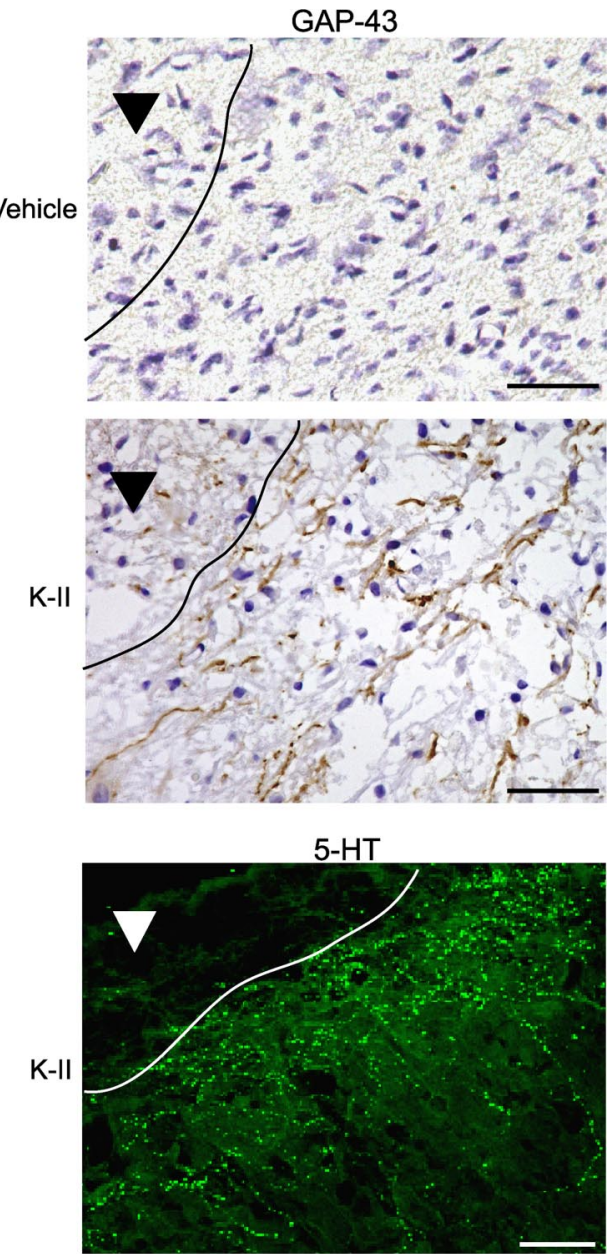

Vehicle $\quad 10 \mathrm{~mm}$ caudal from epicenter

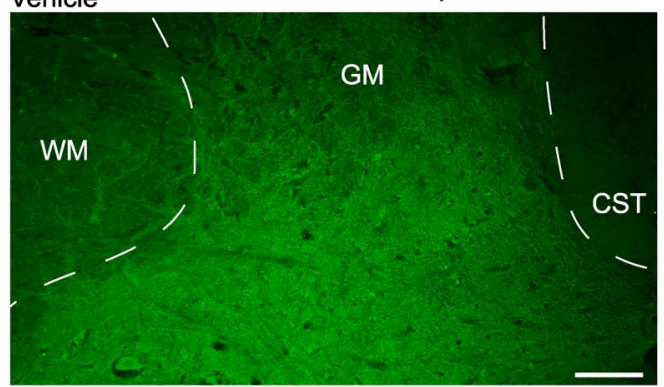

$\mathrm{K}-\mathrm{II}$

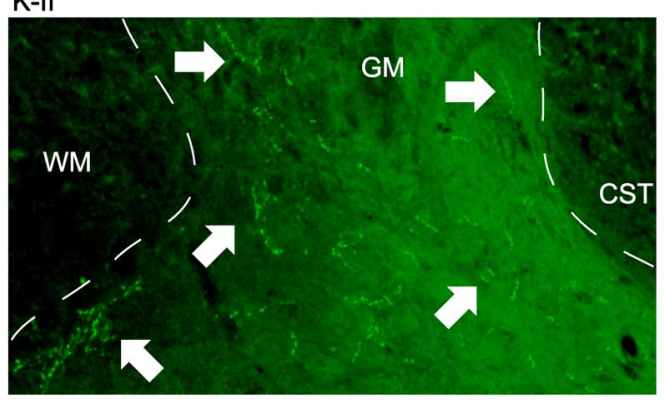

Figure 4. Histological analysis. $A$, The scheme of the methods to reconstruct serial sagittal sections. On each section, the number of GAP-43- and 5-HT-positive fibers was counted from 4 mm rostral to $4 \mathrm{~mm}$ caudal the lesion site. The number was calculated as a percentage of the fibers seen in the sham-operated spinal cord. All serial sections were evaluated at 10 weeks after $\mathrm{SCl}$. $\boldsymbol{B}$, Reconstruction of serial parasagittal section of the number of GAP-43- and 5-HT-positive fibers, represented as a percentage of the fibers seen in the sham-operated spinal cord. Data represent the means \pm SEM. ${ }^{*} p<0.05 ;{ }^{* *} p<0.01 ;{ }^{* * *} p<0.005 ;{ }^{* * *} p<0.001$ versus the vehicle control ( $n=5$ Vehicle; $n=5$ C-ABC; $\left.n=6 \mathrm{~K}-\mathrm{II}\right)$. C, Immunohistochemical analysis of GAP-43 and 5 -HT (serotonin) expression in the injured spinal cord of the boxed area in scheme $A$ at 10 weeks after SCI. Scale bars, $50 \mu \mathrm{m}$ for the panels of GAP-43; $100 \mu \mathrm{m}$ for the panel of 5 -HT. All images are of sagittal section with the left side rostral. Arrowheads indicate the contusion area. D, BDA labeling of CST. The top graph represents the number of labeled corticospinal (Figure legend continues.) 
in the spinal cord. The injury was so severe that the lesion core, which was filled with collagen IV 1 week after injury, occupied a significant area of the spinal cord (data not shown). The injury sizes estimated by collagen IV or GFAP staining did not vary between individual rats (data not shown).

Disulfated KS contents in the injured region increased after injury, peaking at $7 \mathrm{~d}$ (Fig. $1 \mathrm{~A}$ ). CS-C contents also increased, peaking at $\sim 7 \mathrm{~d}$ after injury (Fig. $1 B$ ), consistent with a previous report (Properzi et al., 2005).

$\mathrm{K}-\mathrm{II}(0.05 \mathrm{U})$ or C-ABC $(0.05 \mathrm{U})$ was then locally administered to the injured site for 2 weeks after contusion injuries using an osmotic pump. Disulfated KS contents decreased to the noninjury level after K-II treatment (Fig. 1A). Western blot and immunohistochemical analyses revealed that the reactivity to KS antibody 5D4 was lost in rats treated with K-II for $7 \mathrm{~d}$ after injury, but not in C-ABC-treated rats (Fig. $1 C, D$ ). The smear appearance in Western blot is probably due to the long sugar chains, and is the nature of proteoglycans. The granular staining profile in Figure $1 D$ represented the main source of KS expression, i.e., microglia (Fig. $1 E$ ), which is consistent with previous reports (Jones and Tuszynski, 2002; Ito et al., 2010). As disulfated KS was still detected after K-II treatment by disaccharide analysis using HPLC (Fig. 1B), the almost complete disappearance of KS in Western blot and immunohistochemistry may be due to the limited ability of the 5D4 antibody to detect KS.

To detect diffusion of infused enzyme, biotin-labeled K-II was infused and detected by immunohistochemistry. We found that the enzyme diffused to cover the most area of injury, but did not cover the whole spinal cord (supplemental Fig. 2, available at www.jneurosci.org as supplemental material). These data collectively demonstrated that K-II worked well in vivo, although it did not completely abolish KS.

The in vivo degradation of CS by C-ABC, but not that by K-II, was also confirmed by HPLC analysis (Fig. $1 B$ ) and Western blot analysis (Fig. 1C).

\section{Motor function recovery by K-II}

We next evaluated motor function recovery using the BBB score and \%grip test. K-II led to a striking recovery of motor function; the effect was similar to that of C-ABC (Fig. 2A,B; supplemental Tables 1, 2, available at www.jneurosci.org as supplemental material). There was no significant difference in motor function between the K-II- and C-ABC-treated groups (Fig. $2 A, B$ ). The observed motor function recovery was confirmed by an electrophysiological technique, motor-evoked potential (MEP) monitoring, in which an electric stimulus is given at the thoracic level (Th7), and a response is taken at the gastrocnemius muscles (Fig. $2 C$; supplemental Table 3 , available at www.jneurosci.org as supplemental material). K-II also showed significant functional recovery after SCI even at lower doses, e.g., as low as $2000 \times$ dilution

\footnotetext{
$\leftarrow$

(Figure legend continued.) axons $10 \mathrm{~mm}$ rostral to the lesioned site. No significant difference was observed between treatment groups. The bottom graph represents the ratio of labeled corticospinal axons 4 and $10 \mathrm{~mm}$ caudal versus $10 \mathrm{~mm}$ rostral to the lesioned site. K-II-treated rats had significantly more BDA-positive fibers at 4 and $10 \mathrm{~mm}$ caudal to the lesioned site than vehicle-treated rats. Data represent the means \pm SEM. ${ }^{*} p<0.05$; ${ }^{* *} p<0.005$ versus the vehicle control $(n=7$ Vehicle; $n=6 C-A B C ; n=5 \mathrm{~K}-I I)$. $E$, Representative axial sections of the spinal cord taken from $10 \mathrm{~mm}$ caudal from the lesioned site at 10 weeks after $\mathrm{SCl}$. The boxed areas are magnified on the right side of the panel. BDA-positive fibers were clearly seen in the section of K-II-treated rat (arrows). Scale bars, $500 \mu \mathrm{m}$ for the left panel; $100 \mu \mathrm{m}$ for the right panels. The thin silicone tube of an osmotic mini-pump ${ }^{*}$ ) remained at a point $10 \mathrm{~mm}$ caudal from the epicenter. WM, White matter; GM, gray matter.
}

(Fig. 2D; supplemental Table 4, available at www.jneurosci.org as supplemental material). No significant differences were observed between the K-II-, K-II (10× dilution)-, and K-II (2000× dilution)-treated groups (Fig. 2D). Consistent with this finding, K-II and K-II (2000× dilution) decreased disulfated KS contents in the spinal cord to similar extents (Fig. 1A). This functional recovery was due to the enzymatic activity of K-II, as heatinactivated K-II did not promote motor function recovery at all (Fig. 2 E; supplemental Table 4, available at www.jneurosci.org as supplemental material).

Interestingly, even though $\mathrm{K}-\mathrm{II}$ or $\mathrm{C}-\mathrm{ABC}$ treatment promoted the recovery of motor function, the combination of these two enzymes showed neither additive nor synergistic effects (Fig. $2 C, F$; supplemental Tables 3,5 , available at www.jneurosci.org as supplemental material).

\section{Sensory function recovery by K-II}

SCI induced thermal hyperalgesia and mechanical allodynia (Fig. $3 A, B)$. The tail-immersion test and touch test showed that thermal hyperalgesia and mechanical allodynia were ameliorated by $\mathrm{K}-\mathrm{II}$ or C-ABC treatment, reaching an almost normal level 10 weeks after injury (Fig. 3A,B). sp-SCEP, in which an epidural electric stimulus was given at the Th11 level and a response was taken at the Th7 level, was performed 8 weeks after injury. The results of this test support the idea that the sensory function was recovered by $\mathrm{K}-\mathrm{II}$ or $\mathrm{C}-\mathrm{ABC}$ treatment (Fig. $3 \mathrm{C}$ ). The reinjury of $\mathrm{K}-\mathrm{II}-$ treated rats lost the recovered response of sp-SCEP (Fig. $3 C$ ), and the results were consistent with those of previous reports (Rapalino et al., 1998; Bradbury et al., 2002).

As seen in motor function recovery, the combination of K-II and $\mathrm{C}-\mathrm{ABC}$ showed neither additive nor synergistic effects on sensory function recovery (Fig. $3 C, D$ ).

\section{Histological analysis of functional recovery after SCI}

We reconstructed the serial section of the spinal cord using all of the 50-60 serial longitudinal sections per rat and estimated the number of fibers strained for GAP-43 or 5-HT. The GAP-43 and 5-HT data from the vehicle and treated groups were compared with sham-lesioned controls and expressed as a percentage along the longitudinal axis of the spinal cord (Fig. $4 A, B$ ). We found that GAP-43 staining was significantly enhanced in both the rostral and caudal regions in $\mathrm{K}-\mathrm{II}$ or $\mathrm{C}-\mathrm{ABC}$-treated rats compared with vehicle control rats (Fig. $4 B$ ). The raphespinal tract is partly responsible for motor function in rodents, and consists of serotonin (5-HT)-positive fibers. 5-HT staining showed a striking decrease in 5-HT-positive fibers in the rostral region, especially in vehicle control rats, but $\mathrm{K}-\mathrm{II}$ and $\mathrm{C}-\mathrm{ABC}$ significantly increased the number of stained fibers (Fig. $4 B$ ). On the other hand, in the caudal region, vehicle controls showed almost no stained fibers, whereas $\mathrm{K}-\mathrm{II}$ and $\mathrm{C}-\mathrm{ABC}$ significantly increased the number of stained fibers (Fig. 4B). Representative photos of GAP-45 and $5-\mathrm{HT}$ staining around the lesion were presented in Figure 4C. These results are consistent with the idea that K-II or C-ABC significantly promoted axonal regrowth after SCI.

Furthermore, we performed anterograde labeling of CST to evaluate axonal growth and sprouting. In tracer fiber counts for the CST, we found many fibers stained in the region rostral to the epicenter (supplemental Fig. 3, available at www.jneurosci.org as supplemental material). There was no difference among the three groups in the rostral region (Fig. 4D). BDA-positive fibers were barely detectable in the caudal region of the vehicle group (Fig. $4 D, E)$. These findings support the idea that this contusion model almost completely destroyed CST at the epicenter, and thus BDA- 
positive fibers hardly passed through the lesion to the caudal region in the vehicle group. In contrast, in K-II- and C-ABCtreated rats, the number of BDA-positive fibers was significantly increased in the region caudal to the epicenter, particularly in the gray matter in this region (Fig. $4 D, E$ ). These BDA-positive fibers in the gray matter may reflect growth or sprouting of proximal or spared axons.

\section{Restoration of neurite outgrowth by K-II}

Proteoglycans purified from the brains of chicks contain both KS and CS. We previously determined $300 \mathrm{ng} / \mathrm{ml}$ as the appropriate concentration of proteoglycans to coat dishes, which did not affect cell attachment but inhibited neurite outgrowth (Ito et al., 2010). Accordingly, the substratum coated with these proteoglycans strongly inhibited neurite outgrowth (Fig. 5A, $B$ ), whereas cell binding to the substratum was comparable between conditions tested (Fig. $5 A$ ). The proteoglycan-mediated inhibition of neurite growth was blocked by K-II and $\mathrm{C}-\mathrm{ABC}$, and the effects of $\mathrm{K}-\mathrm{II}$ and $\mathrm{C}-\mathrm{ABC}$ were comparable to each other, and also consistent with our previous study (Ito et al., 2010). To our surprise, the combination of $\mathrm{K}-\mathrm{II}$ and $\mathrm{C}-\mathrm{ABC}$ showed neither synergistic nor additive effects (Fig. 5B). Thus, these in vitro results were consistent with the in vivo phenomena. We also found that heatdenatured proteoglycans as well as reduced/ alkylated proteoglycans lost their inhibitory activity (Fig. 5C), suggesting that the conformation of the core protein structure is also essential to this inhibition, since proteins rather than sugar chains are labile to heat or reduction/alkylation.

As K-II digests KS into oligosaccharides that are each composed of two to four saccharides, we addressed whether or not these degradation products of KS reversed the proteoglycan-mediated inhibition of neurite growth. However, KS-derived oligosaccharides (Fig. 5D) showed no promotion of neurite growth (Fig. $5 E$ ). These results exclude the possibility that the K-II-induced degradation products of KS promote neurite outgrowth, and suggest that K-II directly blocks the inhibition of neurite outgrowth by proteoglycans.

Neurite growth inhibition by KS/CSPG Based on these in vivo and in vitro results, we hypothesized that K-II and C-ABC work on the same axis. We chose aggrecan as a model molecule, as it harbors both KS and CS chains (Fig. 6A) and is a representative proteoglycan in the CNS. Aggrecan strikingly inhibited axonal outgrowth
A
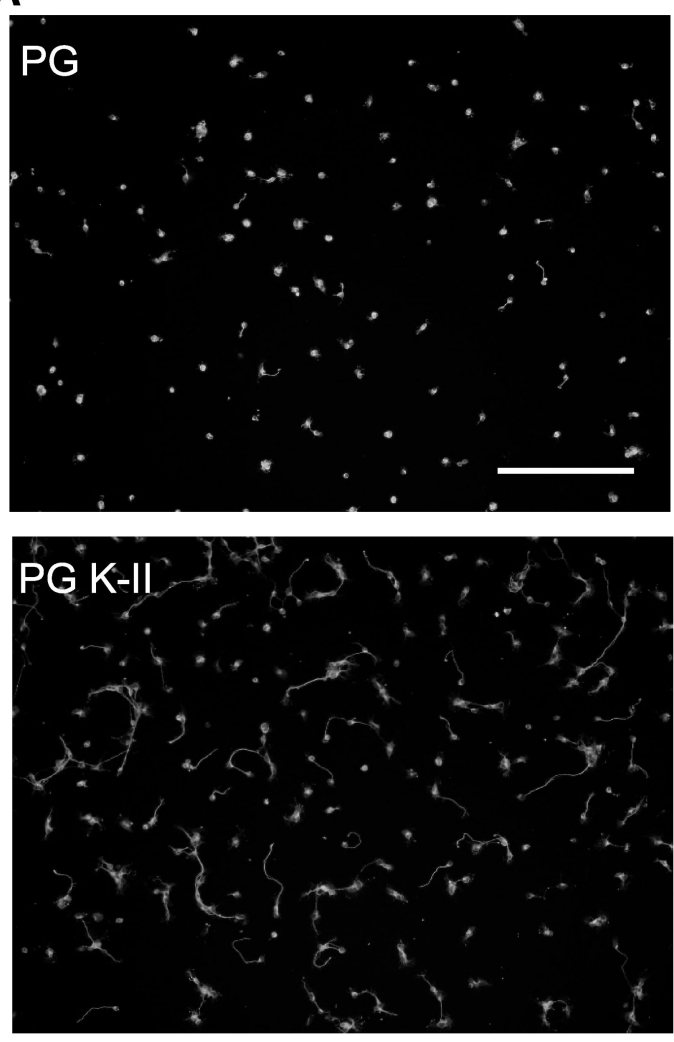

B

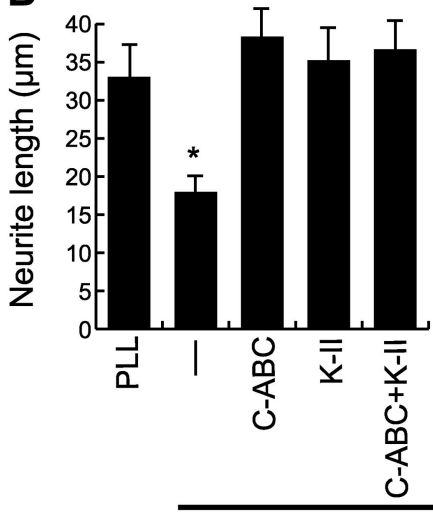

PG

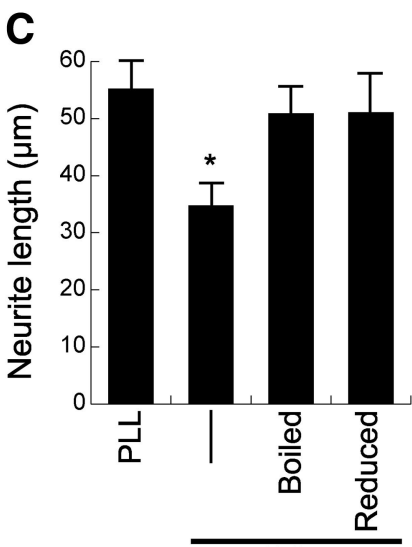

PG

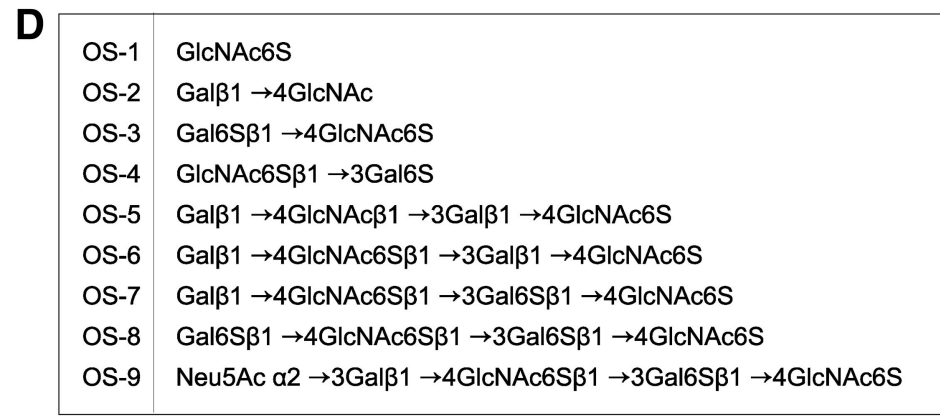

E

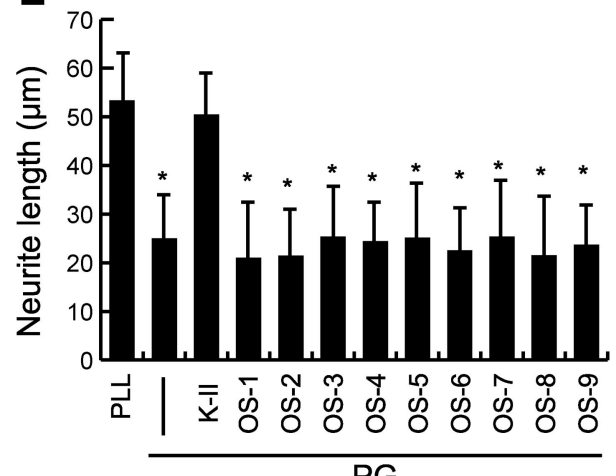

PG

Figure 5. Restoration of neurite outgrowth by K-II. $\boldsymbol{A}, \mathrm{P} 8$ rat cerebellar granular neurons were cultured on proteoglycan (PG) extracted from chick brains. K-II treatment restored neurite outgrowth (PG K-II). Scale bar, $100 \mu \mathrm{m}$. $\boldsymbol{B}$, The quantification of $\boldsymbol{A}$. ${ }^{*} p<0.05$ versus PLL. PG $(300 \mathrm{ng} / \mathrm{ml}), C-A B C(200 \mathrm{mU} / \mathrm{ml})$, and $\mathrm{K}-\mathrm{II}(5 \mathrm{mU} / \mathrm{ml})$ were used. Data represent the average neurite length $\pm S D$. C, Heat-denatured PG lost its neurite outgrowth-inhibitory activity. Data represent the average neurite length $\pm S D$. ${ }^{*} p<0.05$ versus PLL. D, Structures of the oligosaccharides used in this experiment. $\boldsymbol{E}$, P8 rat cerebellar granular neurons were cultured on chick brain PG substrate in the absence or presence of oligosaccharides $(1 \mu \mathrm{g} / \mathrm{ml})$. Data represent the average neurite lengths $\pm S D .{ }^{*} p<0.05$ versus PLL. 
A

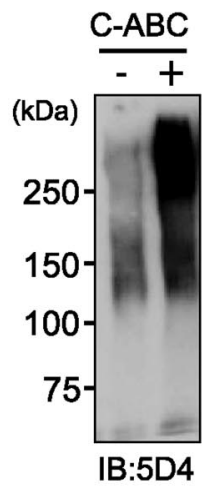

B
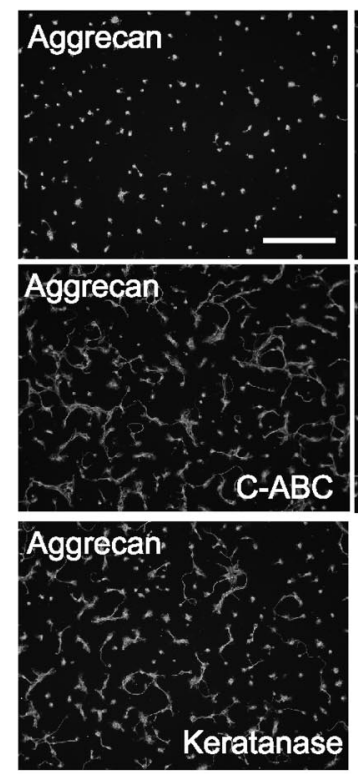

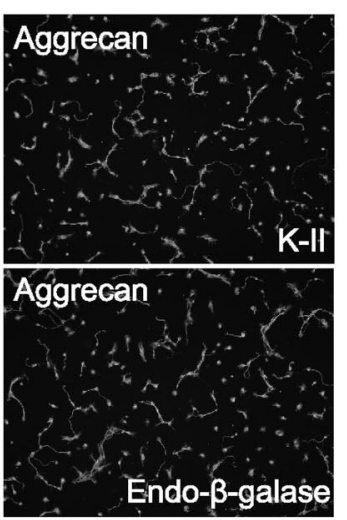

Endo- $\beta$-galase

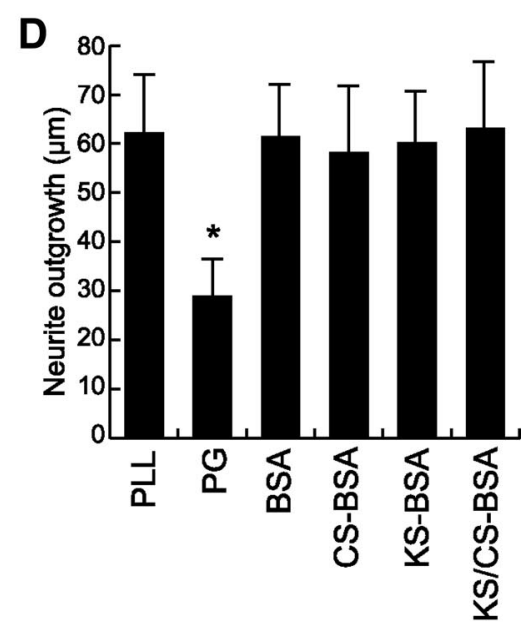

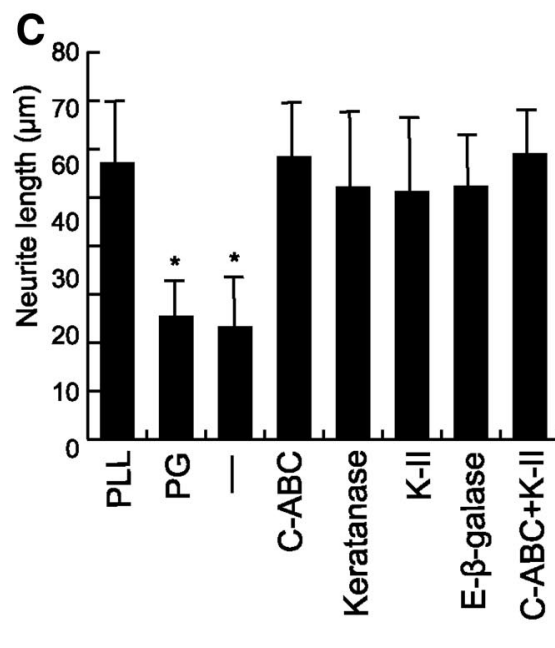

Aggrecan

Figure 6. Effects of KS/CSPGs on neurite outgrowth. $A$, Bovine aggrecan was digested with C-ABC ( $500 \mathrm{mU} / \mathrm{ml})$ and subjected to SDS-PAGE using 5D4. Note that C-ABC treatment exposed KS epitopes. $\boldsymbol{B}$, P8 rat cerebellar granule neurons were cultured on aggrecan $(5 \mu \mathrm{g} / \mathrm{ml})$. Degradation of $C S$ or KS restored neurite outgrowth. Scale bar, $100 \mu \mathrm{m}$. $\boldsymbol{C}$, The quantification of $\boldsymbol{B}$. Data represent the average neurite length $\pm S D .{ }^{*} p<0.05$ versus PLL. $D$, There was no inhibition of neurite outgrowth by CS-BSA, $\mathrm{KS}-\mathrm{BSA}$, or KS/CS-BSA (300 ng/ml each). Data represent the average neurite length \pm SD. ${ }^{*} p<0.05$ versus PLL.

(Fig. 6B,C), consistent with previous studies (Asher et al., 1995; Johnson et al., 2002). The inhibition by aggrecan was as strong as that by a mixture of proteoglycans (Fig. $6 C$ ). Interestingly, this inhibition was reversed by C-ABC as well as K-II. Furthermore, the combination of these enzymes did not show additive or synergistic effects (Fig. 6C).

While K-II digested monosulfated and disulfated KS, keratanase digested monosulfated KS but not disulfated KS. The latter also reversed aggrecan-mediated inhibition of neurite outgrowth (Fig. $6 B, C$ ). Endo- $\beta$-galactosidase, which digests KS and polylactosamine, provided the same effect (Fig. $6 B, C$ ). These results collectively indicate that KS of aggrecan is required for the aggrecan-mediated inhibition of neurite outgrowth.

We then generated an artificial proteoglycan consisting of BSA, KS, and CS. In this experiment, we tried to understand how the three components of KS/CSPG (core protein, CS chains, and KS chains) are needed to exert the inhibitory activity. The esti- mated ratios of the core protein (BSA), CS, and KS in KS/CS-BSA were 1:30:84. Because the ratio in human aggrecan is 1:100:60 (Kiani et al., 2002), KS/CS-BSA was considered suitable for examining the function of KS, CS, and the core protein. However, KS/CS-BSA failed to inhibit neurite outgrowth (Fig. 6D). KS-BSA and CS-BSA also failed to do so (Fig. 6D).

\section{Discussion}

So far, KSPGs and CSPGs have been studied independently, and KSPGs have not been extensively studied. Our study has integrated KS and CS into a common axis. $\mathrm{K}-\mathrm{II}$ and $\mathrm{C}-\mathrm{ABC}$ strikingly promoted functional recovery after SCI. GAP-43, 5-HT staining, and CST tracer fiber count were significantly enhanced in K-II- or C-ABC-treated rats compared with vehicle control rats. Although the striking functional recovery may not be necessarily consistent with the data from the histological analyses, this may have been partly due to the limited ability to detect outgrowing axons by the antibodies and the tracer compound used. We found that the effects of $\mathrm{K}-\mathrm{II}$ and $\mathrm{C}-\mathrm{ABC}$ on in vivo functional disturbance after SCI and in vitro neurite outgrowth inhibition mediated by proteoglycans were comparable to each other. However, both in vivo and in vitro, the combination of these two enzymes showed neither additive nor synergistic effects. It should be noted that the peptide linkage region for CS always consists of xylose, galactose, and glucuronic acid, whereas the KS chain is elongated from ordinary $\mathrm{N}$-linked or $\mathrm{O}$-linked sugar chains. Thus, each glycosaminoglycan exists as an independent sugar chain. For example, human aggrecan is composed of a core protein and CS and KS chains at a ratio of 1:100:60 (Kiani et al., 2002). Together, our study has established that KS and CS are independently required for the inhibition of postinjury neural plasticity and neurite outgrowth.

Furthermore, we found that neurite outgrowth was not inhibited by heat-denatured proteoglycans or by reduced/alkylated proteoglycans. This inhibition was also not achieved by an artificial KS/CSPG, KS/CS-albumin, whereas the natural KS/CSPG aggrecan inhibited neurite outgrowth. Our results thus far obtained are summarized in Figure 7A. Our data suggest that the three components (KS, CS, and core protein) of the proteoglycan moiety are interdependent and required for proteoglycanmediated inhibition of structural rearrangement after neuronal injuries.

It is important to point out that there is a much smaller amount of KS than CS in the CNS, and our data supported this idea (Fig. $1 B, C$ ). The family of KSPGs is also smaller than that of CSPGs (Margolis and Margolis, 1993; Funderburgh, 2000, 2002). In this context, it was surprising to us that K-II shows activity 
A 1. Native PG

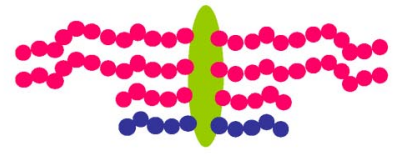

repulsive

2. $\triangle \mathrm{GAGs}$

1

3. Free-GAGs

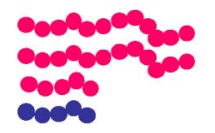

permissive

permissive

permissive

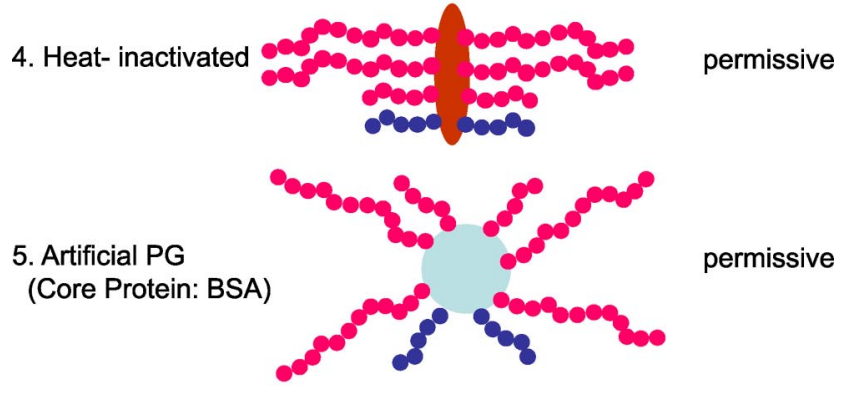

B

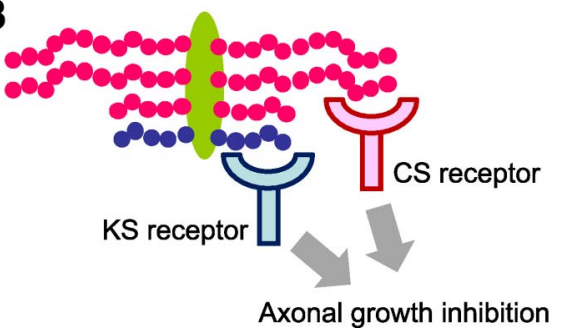

Figure 7. Structure and function relationship in the proteoglycan (PG)-mediated inhibition of neurite growth. $A$, The present study has revealed that $\mathrm{KS}$ and $\mathrm{CS}$ have a comparable impact on PG-mediated inhibition of neurite growth. Considering our previous results that neither KS nor CS is sufficient for neurite growth inhibition (Ito et al., 2010), our data suggest that all three components of the proteoglycans moiety (core protein, $(\mathrm{CS}$, and $\mathrm{KS}$ ) are required for this inhibition activity. $\boldsymbol{B}$, This scheme shows a speculated mechanism underlying the PG-mediated inhibition of axonal growth.

comparable to that of $\mathrm{C}-\mathrm{ABC}$ in vivo and in vitro. Although further investigations are needed, we speculate the following mechanism (summarized in Fig. 7B), based on our observations. First, although KS is composed of repeating disaccharide units, the composition is heterogeneous, i.e., there are highly sulfated disaccharide clusters as well as poorly sulfated disaccharide clusters in a KS chain. Therefore, it is conceivable that there is a functional domain in a KS chain (e.g., a highly sulfated stretch of 6-8 saccharides). This is also the case in a CS chain (there are 4 different sulfation variations in CS-CS-A, -C, -D, and -E units). Second, these functional domains may appear more frequently in KS than in CS. Finally, these functional domains may be recognized independently by specific receptors so that KS and CS are independently required for the inhibition of neurite outgrowth (Fig. 7B). Our idea is partly supported by the recent report that PTP $\sigma$ is the receptor for CS (Shen et al., 2009; Coles et al., 2011). However, there is another possibility. C-ABC might not be very stable, although a significant portion of CS appeared to be lost by C-ABC treatment on Western blot analysis (Fig. 1C). Thus, we should not exclude the possibility that an insufficient in vivo digestion of
CS by our current method led to the lack of synergism of K-II and C-ABC.

Reported data regarding the biological impacts of KS and CS on neurite outgrowth have been contradictory. For example, Powell et al. (1997) reported in vitro data that showed the effects of CS and KS are similar, but that enzymatic treatment with $\mathrm{C}-\mathrm{ABC}$ and keratanase is synergistic. In contrast, Snow et al. (1990b) showed that in vitro enzymatic removal of KS and CS from CSPGs differ with regard to neurite behavior (CS is stronger than KS). Furthermore, in cultured hippocampal slices, keratanase, but not $\mathrm{C}-\mathrm{ABC}$, leads to misrouting of mossy fibers (Butler et al., 2004). In the present study, we conducted experiments both in vivo and in vitro to address the same issue. Our study has revealed that $\mathrm{KS}$ and $\mathrm{CS}$ are independent requirements for the proteoglycan-mediated inhibition of postinjury neural plasticity in vivo. Taking into account that the $\mathrm{K}$-II used for in vivo experiments in the present study is relatively stable at $37^{\circ} \mathrm{C}$ and has a wide window of therapeutic doses, our in vivo data may be reliable. In vitro results were consistent with in vivo data. Although discrepancy between reports might be due to different conditions examined, our results may provide a hint for further studies on molecular mechanisms of the neural plasticity regulation by proteoglycans.

The functional recovery by K-II or C-ABC after SCI may not simply rely on neurite outgrowth promotion by these enzymes. The ECM surrounding neurons forms a lace-like structure, the so-called perineuronal net (Takahashi-Iwanaga et al., 1998), and is essentially composed of hyaluronic acid and proteoglycans. Perineuronal nets are prominent on GABAergic interneurons, but can be detected on virtually all neurons (Celio and Blümcke, 1994; Celio et al., 1998; John et al., 2006). This ECM may inhibit structural rearrangements at synapses, thereby contributing to the maintenance of neuronal networks; this idea is supported by recent works showing that $\mathrm{C}-\mathrm{ABC}$ promotes synaptic plasticity and dendritic spine dynamics (Pizzorusso et al., 2002, 2006; Berardi et al., 2004; Oray et al., 2004). C-ABC restores the plasticity of not only sensory networks but also emotional networks (Pizzorusso et al., 2002; Gogolla et al., 2009). Furthermore, hyaluronidase, a hyaluronic acid-degrading enzyme, and C-ABC increase AMPA receptor lateral mobility, and consequently modulate short-term synaptic plasticity (Frischknecht et al., 2009). Considering these data together, our results suggest that $\mathrm{K}-\mathrm{II}$ and $\mathrm{C}-\mathrm{ABC}$ may remove the ECM and promote structural rearrangement of neuronal networks after SCI. In this context, it is an interesting challenge to ask whether K-II also restores ocular dominance plasticity in adults.

Regarding the lateral mobility of membrane molecules such as AMPA receptor on dendrites, the ECM works as a viscous diffusion constraint that slows and confines the mobility of molecules entering the ECM-covered compartments (Frischknecht et al., 2009). Thus, a general function of the ECM is to act as a passive barrier. On the other hand, it has recently been reported that $\mathrm{PTP} \sigma$ acts as a receptor for CS and mediates the inhibitory activity of CSPGs on axonal regeneration (Shen et al., 2009). Our data raise the intriguing possibility that a KS-specific receptor may also exist (Fig. $7 B$ ).

It is also important to consider that KS degradation within the intact area may cause maladaptive plasticity if K-II affects the ECM structure. However, as shown in Figure 3, K-II administration promoted recovery from thermal hyperalgesia and mechanical allodynia. Thus, the delivery method used in our study could minimize the unwanted adverse effect of KS degradation. Supporting this idea, we observed that in vivo KS degradation by K-II 
was good enough to promote functional recovery, but not completely abolish KS, probably due to a limited diffusion of infused enzyme.

In addition to the therapeutic effects of K-II on SCI, we also noticed that the functional recovery induced by K-II or C-ABC was not complete. Therefore, further improvement of a therapeutic strategy for SCI must be considered. García-Alías et al. (2009) recently reported that a combination of C-ABC and rehabilitation leads to a striking functional recovery after SCI at the cervical level. Even if our present results suggest that K-II or $\mathrm{C}-\mathrm{ABC}$ may promote not only axonal regrowth but also neural plasticity related to motor and sensory function, other efforts, such as rehabilitation, to improve the reestablishment of neural circuitry would be helpful to realize a satisfactory treatment for SCI.

\section{References}

Asher RA, Scheibe RJ, Keiser HD, Bignami A (1995) On the existence of a cartilage-like proteoglycan and link proteins in the central nervous system. Glia 13:294-308.

Basso DM, Beattie MS, Bresnahan JC (1995) A sensitive and reliable locomotor rating scale for open field testing in rats. J Neurotrauma 12:1-21.

Berardi N, Pizzorusso T, Maffei L (2004) Extracellular matrix and visual cortical plasticity: freeing the synapse. Neuron 44:905-908.

Bradbury EJ, Moon LD, Popat RJ, King VR, Bennett GS, Patel PN, Fawcett JW, McMahon SB (2002) Chondroitinase ABC promotes functional recovery after spinal cord injury. Nature 416:636-640.

Butler CD, Schnetz SA, Yu EY, Davis JB, Temple K, Silver J, Malouf AT (2004) Keratan sulfate proteoglycan phosphacan regulates mossy fiber outgrowth and regeneration. J Neurosci 24:462-473.

Celio MR, Blümcke I (1994) Perineuronal nets-a specialized form of extracellular matrix in the adult nervous system. Brain Res Brain Res Rev 19:128-145.

Celio MR, Spreafico R, De Biasi S, Vitellaro-Zuccarello L (1998) Perineuronal nets: past and present. Trends Neurosci 21:510-515.

Cole GJ, McCabe CF (1991) Identification of a developmentally regulated keratan sulfate proteoglycan that inhibits cell adhesion and neurite outgrowth. Neuron 7:1007-1018.

Coles CH, Shen Y, Tenney AP, Siebold C, Sutton GC, Lu W, Gallagher JT, Jones EY, Flanagan JG, Aricescu AR (2011) Proteoglycan-specific molecular switch for RPTP $\sigma$ clustering and neuronal extension. Science 332:484-488.

Davies SJ, Goucher DR, Doller C, Silver J (1999) Robust regeneration of adult sensory axons in degenerating white matter of the adult rat spinal cord. J Neurosci 19:5810-5822.

Frischknecht R, Heine M, Perrais D, Seidenbecher CI, Choquet D, Gundelfinger ED (2009) Brain extracellular matrix affects AMPA receptor lateral mobility and short-term synaptic plasticity. Nat Neurosci 12:897-904.

Funderburgh JL (2000) Keratan sulfate: structure, biosynthesis, and function. Glycobiology 10:951-958.

Funderburgh JL (2002) Keratan sulfate biosynthesis. IUBMB Life 54:187-194.

García-Alías G, Barkhuysen S, Buckle M, Fawcett JW (2009) Chondroitinase $A B C$ treatment opens a window of opportunity for task-specific rehabilitation. Nat Neurosci 12:1145-1151.

Geisert EE Jr, Bidanset DJ, Del Mar N, Robson JA (1996) Up-regulation of a keratan sulfate proteoglycan following cortical injury in neonatal rats. Int J Dev Neurosci 14:257-267.

Gogolla N, Caroni P, Lüthi A, Herry C (2009) Perineuronal nets protect fear memories from erasure. Science 325:1258-1261.

Grimpe B, Silver J (2004) A novel DNA enzyme reduces glycosaminoglycan chains in the glial scar and allows microtransplanted dorsal root ganglia axons to regenerate beyond lesions in the spinal cord. J Neurosci 24:1393-1397.

Hutchinson KJ, Gómez-Pinilla F, Crowe MJ, Ying Z, Basso DM (2004) Three exercise paradigms differentially improve sensory recovery after spinal cord contusion in rats. Brain 127:1403-1414.

Ito Z, Sakamoto K, Imagama S, Matsuyama Y, Zhang H, Hirano K, Ando K, Yamashita T, Ishiguro N, Kadomatsu K (2010) N-acetylglucosamine
6-O-sulfotransferase-1-deficient mice show better functional recovery after spinal cord injury. J Neurosci 30:5937-5947.

John N, Krügel H, Frischknecht R, Smalla KH, Schultz C, Kreutz MR, Gundelfinger ED, Seidenbecher CI (2006) Brevican-containing perineuronal nets of extracellular matrix in dissociated hippocampal primary cultures. Mol Cell Neurosci 31:774-784.

Johnson WE, Caterson B, Eisenstein SM, Hynds DL, Snow DM, Roberts S (2002) Human intervertebral disc aggrecan inhibits nerve growth in vitro. Arthritis Rheum 46:2658-2664.

Jones LL, Tuszynski MH (2002) Spinal cord injury elicits expression of keratan sulfate proteoglycans by macrophages, reactive microglia, and oligodendrocyte progenitors. J Neurosci 22:4611-4624.

Kiani C, Chen L, Wu YJ, Yee AJ, Yang BB (2002) Structure and function of aggrecan. Cell Res 12:19-32.

Krautstrunk M, Scholtes F, Martin D, Schoenen J, Schmitt AB, Plate D, Nacimiento W, Noth J, Brook GA (2002) Increased expression of the putative axon growth-repulsive extracellular matrix molecule, keratan sulphate proteoglycan, following traumatic injury of the adult rat spinal cord. Acta Neuropathol 104:592-600.

Margolis RK, Margolis RU (1993) Nervous tissue proteoglycans. Experientia 49:429-446.

Massey JM, Hubscher CH, Wagoner MR, Decker JA, Amps J, Silver J, Onifer SM (2006) Chondroitinase $\mathrm{ABC}$ digestion of the perineuronal net promotes functional collateral sprouting in the cuneate nucleus after cervical spinal cord injury. J Neurosci 26:4406-4414.

Moon LD, Asher RA, Rhodes KE, Fawcett JW (2001) Regeneration of CNS axons back to their target following treatment of adult rat brain with chondroitinase ABC. Nat Neurosci 4:465-466.

Moon LD, Asher RA, Rhodes KE, Fawcett JW (2002) Relationship between sprouting axons, proteoglycans and glial cells following unilateral nigrostriatal axotomy in the adult rat. Neuroscience 109:101-117.

Oray S, Majewska A, Sur M (2004) Dendritic spine dynamics are regulated by monocular deprivation and extracellular matrix degradation. Neuron 44:1021-1030.

Pizzorusso T, Medini P, Berardi N, Chierzi S, Fawcett JW, Maffei L (2002) Reactivation of ocular dominance plasticity in the adult visual cortex. Science 298:1248-1251.

Pizzorusso T, Medini P, Landi S, Baldini S, Berardi N, Maffei L (2006) Structural and functional recovery from early monocular deprivation in adult rats. Proc Natl Acad Sci U S A 103:8517-8522.

Powell EM, Fawcett JW, Geller HM (1997) Proteoglycans provide neurite guidance at an astrocyte boundary. Mol Cell Neurosci 10:27-42.

Properzi F, Carulli D, Asher RA, Muir E, Camargo LM, van Kuppevelt TH, ten Dam GB, Furukawa Y, Mikami T, Sugahara K, Toida T, Geller HM, Fawcett JW (2005) Chondroitin 6-sulphate synthesis is up-regulated in injured CNS, induced by injury-related cytokines and enhanced in axongrowth inhibitory glia. Eur J Neurosci 21:378-390.

Pumphrey CY, Theus AM, Li S, Parrish RS, Sanderson RD (2002) Neoglycans, carbodiimide-modified glycosaminoglycans: a new class of anticancer agents that inhibit cancer cell proliferation and induce apoptosis. Cancer Res 62:3722-3728.

Rapalino O, Lazarov-Spiegler O, Agranov E, Velan GJ, Yoles E, Fraidakis M, Solomon A, Gepstein R, Katz A, Belkin M, Hadani M, Schwartz M (1998) Implantation of stimulated homologous macrophages results in partial recovery of paraplegic rats. Nat Med 4:814-821.

Shen Y, Tenney AP, Busch SA, Horn KP, Cuascut FX, Liu K, He Z, Silver J, Flanagan JG (2009) PTP $\sigma$ is a receptor for chondroitin sulfate proteoglycan, an inhibitor of neural regeneration. Science 326:592-596.

Shinmei M, Miyauchi S, Machida A, Miyazaki K (1992) Quantitation of chondroitin 4-sulfate and chondroitin 6-sulfate in pathologic joint fluid. Arthritis Rheum 35:1304-1308.

Silver J, Miller JH (2004) Regeneration beyond the glial scar. Nat Rev Neurosci 5:146-156.

Sivasankaran R, Pei J, Wang KC, Zhang YP, Shields CB, Xu XM, He Z (2004) PKC mediates inhibitory effects of myelin and chondroitin sulfate proteoglycans on axonal regeneration. Nat Neurosci 7:261-268.

Snow DM, Steindler DA, Silver J (1990a) Molecular and cellular characterization of the glial roof plate of the spinal cord and optic tectum: a possible role for a proteoglycan in the development of an axon barrier. Dev Biol 138:359-376.

Snow DM, Lemmon V, Carrino DA, Caplan AI, Silver J (1990b) Sulfated 
proteoglycans in astroglial barriers inhibit neurite outgrowth in vitro. Exp Neurol 109:111-130.

Sutter M, Deletis V, Dvorak J, Eggspuehler A, Grob D, Macdonald D, Mueller A, Sala F, Tamaki T (2007) Current opinions and recommendations on multimodal intraoperative monitoring during spine surgeries. Eur Spine J 16 [Suppl 2]:S232-S237.

Takahashi-Iwanaga H, Murakami T, Abe K (1998) Three-dimensional microanatomy of perineuronal proteoglycan nets enveloping motor neurons in the rat spinal cord. J Neurocytol 27:817-827.

Tamaki T, Kubota S (2007) History of the development of intraoperative spinal cord monitoring. Eur Spine J 16 [Suppl 2]:S140-S146.

Tom VJ, Steinmetz MP, Miller JH, Doller CM, Silver J (2004) Studies on the development and behavior of the dystrophic growth cone, the hallmark of regeneration failure, in an in vitro model of the glial scar and after spinal cord injury. J Neurosci 24:6531-6539.

Yamagishi K, Suzuki K, Imai K, Mochizuki H, Morikawa K, Kyogashima M, Kimata K, Watanabe H (2003) Purification, characterization, and molecular cloning of a novel keratan sulfate hydrolase, endo- $\beta-N$ acetylglucosaminidase, from Bacillus circulans. J Biol Chem 278: $25766-25772$.

Zhang H, Muramatsu T, Murase A, Yuasa S, Uchimura K, Kadomatsu K (2006) $\mathrm{N}$-Acetylglucosamine 6-O-sulfotransferase-1 is required for brain keratan sulfate biosynthesis and glial scar formation after brain injury. Glycobiology 16:702-710. 